LBNL-42973

SCMAG-660

\title{
Bent Superconducting Solenoids for the Muon Cooling Experiment
}

\author{
M. A. Green, \\ Lawrence Berkeley National Laboratory \\ University of California \\ Berkeley CA 94720 \\ Y. Eyssa, S. Kenney, J. R. Miller, S. Prestemon \\ National High Magnetic Field Laboratory \\ Florida State University \\ Tallahassee FL 32310 \\ S. T. Wang, \\ Wang NMR Incorporated \\ Livermore CA 94550
}

March 1999

1999 Particle Accelerator Conference

New York NY, 29 March to 2 April 1999

To be Published in the PAC-99 Proceedings

* This work was supported by the Office of High Energy and Nuclear Physics United States Department of Energy under contract number DE-AC03-76SF00098. 


\title{
Bent Superconducting Solenoids for the Muon Cooling Experiment*
}

\author{
Y. Eyssa**, M. A. Green*+, S. Kenney**, J. R. Miller**, S. Prestemon**, S. T. Wang*** \\ *Lawrence Berkeley National Laboratory, University of California, Berkeley CA 94720 \\ **National High Magnetic Field Laboratory, Florida State University, Tallahassee FL 32310 \\ **Wang NMR Inc., Livermore CA 94550
}

\section{Abstract}

This report describes some solenoid design work done for the cooling experiment for the muon collider collaboration. This report describes an analysis section of superconducting solenoids that have a center line induction of $3.0 \mathrm{~T}$. The section is bent in the shape of an S. Each bend in the $S$ bends the muon beam one radian ( 57.3 degrees). The warm bore diameter of the solenoid bent solenoid is 300 to $320 \mathrm{~mm}$. The radius of the bend at the solenoid center line is $1000 \mathrm{~mm}$. This report shows the results of three dimensional field calculations and presents a solenoid design that will include four TPC detectors that are $240 \mathrm{~mm}$ in diameter and $550 \mathrm{~mm}$ long as well as a $1300 \mathrm{~mm}$ long section of $1300 \mathrm{MHz}$ RF cavities. The TPC sections need a solenoid warm bore diameter of about $300320 \mathrm{~mm}$ while RF cavities require a warm bore diameter of $440 \mathrm{~mm}$. The superconducting solenoid design must take into account the varying warm bore diameter requirements for the magnet string yet meet the stringent solenoidal field uniformity requirements within the active volume of the four TPCs.

\section{BACKGROUND}

The proposed muon cooling experiment $[1,2]$ consists of a pair of $\mathrm{S}$ shaped solenoid bends each of which has two bent solenoids, and straight solenoids for four TPC detectors and an $805 \mathrm{MHz}$ RF cavity[3]. Between the two $S$ shaped bend sections is a straight muon cooling section. The energy and momentum of the muon beam are analyzed by the $\mathrm{S}$ shaped bent solenoid systems before and after the straight solenoidal muon cooling section.

After the beam first analysis, the muon beam enters a muon cooling section that is about 15 meter long. The cooling section consists of ten alternating solenoids with a peak induction of $15 \mathrm{~T}$. The muons are cooled by a liquid hydrogen section that is about $400 \mathrm{~mm}$ long and $70 \mathrm{~mm}$ in diameter. After the muon momentum has been reduced by about $25 \mathrm{MeV} / \mathrm{c}$, the muon are re accelerated by a 900 $\mathrm{mm}$ long $805 \mathrm{MHz}$ RF cavity section back to their original energy of about $200 \mathrm{MeV}$. The second S shaped solenoid section analyzes the energy and momentum of the muons after they have gone through cooling.

The bent solenoid separates muons by momentum spatially across the bore of the solenoid following the bent solenoid. A coil that generates a pure dipole field in the bent solenoid moves the momentum separated muons so that their momentum separation is distributed around the center of the solenoid following the bent solenoid.

\footnotetext{
* This work was supported by the Office of High Energy and Nuclear Physics United States Department of Energy under contract number DE-AC03-76SF00098.

+ email: magreen.lbl.gov
}

\section{THE BENT SOLENOID DESIGN}

The following design assumptions were used for the preliminary design of the superconducting bent solenoid system: 1) Magnetic flux is conserved in the solenoids. Magnetic flux conservation means that all of the solenoid coils have the same average current radius. 2) The warm bore diameter for the for the two TPC solenoids and the bent solenoid is $320 \mathrm{~mm}$. The solenoid around the RF cavity has a warm bore diameter of $440 \mathrm{~mm}$. 3) The bend angle for the bent solenoid is 57.3 degrees ( 1 radian). The bend radius for the bent solenoid axis is $1000 \mathrm{~mm}$. 4) The bent solenoid induction on axis is $3.0 \mathrm{~T}$. The bent solenoid dipole is set at $0.51 \mathrm{~T}$ on the axis. The dipole windings are assumed to be separately powered from the solenoid windings in the bent solenoid. The value of the bent solenoid dipole induction can be increased to $0.75 \mathrm{~T}$, if needed. 5) Standard MRI superconductor is assumed for the solenoid windings. The solenoids are hooked together electrically in series. 6) In the TPC, the integral of the $r$ component of field over the integral of the $\mathrm{z}$ component of field is less than 0.0002 within the TPC active volume. The TPC active volume is assumed to be $160 \mathrm{~mm}$ in diameter by $500 \mathrm{~mm}$ long near the center of the TPC solenoid. 7) The TPC cables are fed out of the solenoid through room temperature slots between coils that are located at the ends of the TPCs away from the bent solenoid. 8) The two TPC solenoids, the bent solenoid and the RF solenoid share a common cryostat vacuum vessel. The two straight solenoids and the bent solenoid are assumed to have separate cold mass support systems. The cryostat ends at the center of the RF cavity so that the RF wave guide can enter the cavity between the solenoids. 9) Conduction cooling to a system of pipes carrying twophase helium is assumed.

The superconducting magnet system from the first TPC solenoid to the solenoid that covers half of the RF cavity is shown in Figure 1. The average coil diameter for the magnets shown in Figure 1 is $480 \mathrm{~mm}$. The smallest coil diameter in the TPC magnet section is about $450 \mathrm{~mm}$. The coils around the RF coil clear the warm bore of the cryostat by about $10 \mathrm{~mm}$. The coils around the RF cavities are located inside of an aluminum support structure that has the helium cooling tube attached to it. The extra dipole coils for the bent solenoid ate not shown in Fig. 1. The solenoidal coils parameters are shown in Table 1. All of the solenoidal coil shown in Fig. 1 and described in Table 1 are designed to be powered by a single power supply. The total stored energy in the string shown in Fig. 1 is 3.44 MJ. The high current density magnet coils (163.3 A per square $\mathrm{mm}$ ) would require that the coils be subdivided so that they can be protected by cold diodes and resistors. 


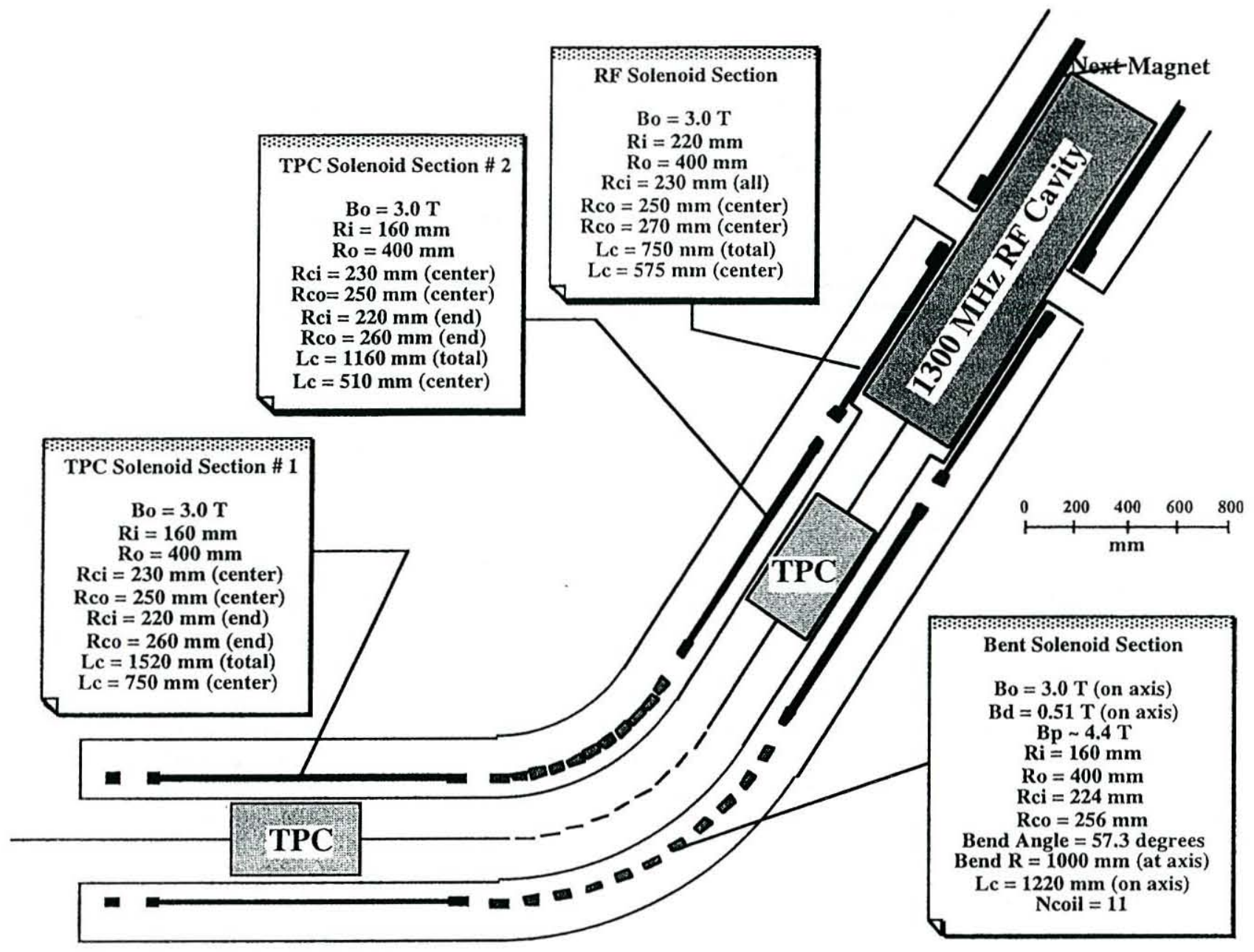

Figure 2: A Cross-section of a 57.3 degree Bent Solenoid section with Straight Solenoid Sections for Half of an RF Cavity and two TPC Detectors

Table 1: Magnet Parameters for the Bent Solenoid System

TPC \# 1 Bent Sol.

Warm Bore Diameter (mm)

Cryostat Outer Diameter (mm)

Cryostat Section Length on axis $(\mathrm{m})$

Coil Average Diameter $(\mathrm{mm})$

Coil Thickness (center) (mm)

Coil Thickness (ends) (mm)

Number of layers (center)

Number of Layers (ends)

Central Induction on axis $(\mathrm{T})$

Coil Design Current (A)

Coil Section Stored Energy (MJ)

Coil Section Inductance $(\mathrm{H})$

Conductor Current Density (A mm-2)

EJ $\left(\mathrm{J} \mathrm{A}^{2} \mathrm{~m}^{-4}\right)^{*}$

$\begin{array}{cc}320 & 320 \\ 800 & 800 \\ 1.7 & 1.2 \\ 480 & 480 \\ 17.9 & 25.7 \\ 34.8 & \mathrm{NA} \\ 16 & 23 \\ 32 & \mathrm{NA} \\ 3.0 & 3.0 \\ 257.3 & 257.3 \\ 1.10 & 0.78 \\ 33.2 & 23.7 \\ 163.3 & 163.3 \\ 2.9 \times 10^{22} & 2.1 \times 10^{22} \\ \end{array}$

TPC \#2

RF Sol.

$\begin{array}{cc}320 & 440 \\ 800 & 800 \\ 1.6 & 0.8 \\ 480 & 480 \\ 17.9 & 17.9 \\ 34.8 & 34.8 \\ 16 & 16 \\ 32 & 32 \\ 3.0 & 3.0 \\ 257.3 & 257.3 \\ 1.04 & 0.52 \\ 31.4 & 15.7 \\ 163.3 & 163.3 \\ 2.8 \times 10^{22} & 1.4 \times 10^{22}\end{array}$

* Quench protection must be supplied to each magnet. Cold diode and resistor quench protection is attractive for these magnets. A separate power supply can be used for each string of magnets. HTS current leads could be used. 


\section{THE BENT DIPOLE}

Figure 1 does not show the dipole coils needed to move the momentum separated muon back to the center of the solenoid. When the muon momentum is $180 \mathrm{MeV} / \mathrm{c}$ and the bent solenoid induction on axis is $3.0 \mathrm{~T}$, a pure dipole of $0.51 \mathrm{~T}$ will center the muon momentum spread about the physical center of the dipole. The dipole must be a pure dipole to do this. At first blush, it appears that mounting a cosine theta current distribution on the outside of the bent solenoid (or just inside the bent solenoid coils) would produce a pure dipole. Unfortunately, it produces a gradient dipole (a pure dipole plus a quadrupole) that is stronger on the inside than on the outside. Redistributing the cosine theta coils so that the current density as a function of angle is the same on the inside of the bend and the outside of the bend will get rid of the gradient. This means that the pole for the dipole is displaced outward away from the axis of the major bend. The displacement angle with respect to a bent plane that passes through the bent axis of the solenoid is only a few degrees. The amount of this angle is dependent on the radius of the dipole with respect to the minor axis.

The ends of the dipole pose a problem in that they introduce local field errors. The integrated dipole field along the minor axis of the bent solenoid due to the ends of the dipole can be made quite good. This is an acceptable solution provided the longitudinal gyration period of the muons in the solenoid is long compared to the radius of the dipole coils.

The dipole coils should be separately powered from the solenoid coils. This will allow the dipole to be adjusted for the average momentum of the muons in the cooling channel. The muon cooling experiment is expected to be done over a range of muon momenta from $160 \mathrm{MeV} / \mathrm{c}$ to $250 \mathrm{MeV} / \mathrm{c}$. An adjustment of the dipole field that is separate from the solenoid field will permit a range of muon momenta to be analyzed. The design peak induction (on the center of the bent solenoid) for the dipole in the bent solenoid could be as high as $0.8 \mathrm{~T}$, whereas the nominal dipole induction is $0.51 \mathrm{~T}$.

\section{SOLENOID FIELD UNIFORMITY}

The field uniformity specification for the straight solenoids is nominally 1 part in 1000 at a $75 \mathrm{~mm}$ radius. The studies that were done were done for solenoids with coils that have a $200 \mathrm{~mm}$ current radius. The field uniformity possible appears to be about 1 part in 2000 within this region. The studies also indicate that a field uniformity of better than 1 part in 1000 is achievable even when there is a $100 \mathrm{~mm}$ gap between coils, provided the ends of the coils are built up to make up for the missing current in the gap.

The field times the radius from the major axis within the curved section is for the most part good to 1 part in 500. The computer model used ten straight coils with an average current radius of $200 \mathrm{~mm}$ to calculate the field uniformity within the bent solenoid. The uniformity of the field times the distance from the major axis improves as the number of coils in the bent solenoid increases, The greatest field non uniformity occurs at the transition from the straight section to the curved section. The pure dipole field can be made uniform to 1 part 1000 except where the ends cross over the solenoidal coils. Moving the current sheet out to a radius of $240 \mathrm{~mm}$ should improve the field uniformity by more than a factor of two.

\section{CONCLUDING COMMENTS}

It appears that a superconducting bent solenoid system can be built for the muon cooling experiment using existing MRI superconductor. The dipole coils can also using MRI superconductor. Conduction cooling of the superconducting coils using two-phase helium in tubes appears to be a good way of cooling these coils.

The field uniformity required for the TPC detectors appears to be achievable in a superconducting solenoid magnet with an average coil radius of $240 \mathrm{~mm}$. The bent solenoid field uniformity (defined as the field uniformity times the distance from the major axis) within a bore that is $75 \mathrm{~mm}$ in radius is also acceptable for a ten coil bent solenoid with an average current radius of $240 \mathrm{~mm}$.

A winding configuration of a pure dipole coil mounted onto the bent solenoid also appears to be possible to fabricate. The major problem with this dipole is the effect of end windings on the field uniformity within both the straight and bent solenoids. For the dipole solution to be acceptable, the integrated dipole field must be uniform and the gyration length for the muons in the solenoid must be long compared to the dipole radius.

\section{ACKNOWLEDGMENTS}

The authors acknowledge discussions with Robert Palmer, Kirk McDonald and others concerning the bent solenoid system for the muon cooling experiment. This work was supported by the Office of High Energy and Nuclear Physics United States Department of Energy under contract number DE-AC03-76SF00098.

\section{REFERENCES}

[1] The Muon Collider Collaboration, "Status of Research, Development and Future Plans," BNL-65-623, Fermeilab-PUB-98/179 and LBNL-41935 (1998)

[2] K. T. McDonald, "Muon Colliders: Status of R\&D and Future Plans," (This Proceedings) the Proceedings of the 1999 Particle Accelerator Conference, New York, NY 29 March to 2 April 1999, IEEE Publications (1999)

[3] S. A. Kahn, H. Guler, C. Lu, K. T. McDonald, E. Prebys and S. E. Vahsen, "The Instrumentation Channel for the MUCOOL Experiment," (This Proceedings) the Proceedings of the 1999 Particle Accelerator Conference, New York, NY 29 March to 2 April 1999, IEEE Publications (1999) 